\title{
Electron trapping and reinjection in prepulse-shaped gas targets for laser-plasma accelerators
}

\author{
R. H. H. Scottఠ, ${ }^{1,}{ }^{*}$ C. Thornton, ${ }^{2}$ N. Bourgeois, ${ }^{2}$ J. Cowley, ${ }^{2}$ Wolf Rittershofer, ${ }^{2}$ \\ Tobias Kleinwächter, ${ }^{3}$ Jens Osterhoff, ${ }^{3}$ D. R. Symes, ${ }^{1}$ C. Hooker, ${ }^{1}$ and S. M. Hooker ${ }^{2}$ \\ ${ }^{1}$ Central Laser Facility, STFC Rutherford Appleton Laboratory, \\ Harwell Oxford, Didcot, OX11 OQX, United Kingdom \\ ${ }^{2}$ Department of Physics and John Adams Institute for Accelerator Science, University of Oxford, \\ Denys Wilkinson Building, Keble Road, Oxford OX1 3RH, United Kingdom \\ ${ }^{3}$ DESY, Notkestr 85, 22607 Hamburg, Germany
}

(Received 2 January 2019; revised 27 February 2020; accepted 23 September 2020; published 11 November 2020)

\begin{abstract}
A novel mechanism for injection, emittance selection, and postacceleration for laser wakefield electron acceleration is identified and described. It is shown that a laser prepulse can create an ionized plasma filament through multiphoton ionization and this heats the electrons and ions, driving an ellipsoidal blast-wave aligned with the laser-axis. The subsequent high-intensity laser-pulse generates a plasma wakefield which, on entering the leading edge of the blast-wave structure, encounters a sharp reduction in electron density, causing density down-ramp electron injection. The injected electrons are accelerated to $\sim 2 \mathrm{MeV}$ within the blast-wave. After the main laser-pulse has propagated past the blast-wave, it drives a secondary wakefield within the homogenous background plasma. On exiting the blast-wave structure, the preaccelerated electrons encounter these secondary wakefields, are retrapped, and accelerated to higher energies. Due to the longitudinal extent of the blast-wave, only those electrons with small transverse velocity are retrapped, leading to the potential for the generation of electron bunches with reduced transverse size and emittance.
\end{abstract}

DOI: 10.1103/PhysRevAccelBeams.23.111301

\section{INTRODUCTION}

Laser wakefield acceleration of quasi-monoenergetic relativistic electron bunches [1-3] have the potential to reduce the scale, and thus cost, of future particleaccelerators as the accelerating fields are 3-4 orders of magnitude higher than those achievable in state-of-the-art radio-frequency accelerators.

Key challenges for the LWFA scheme are electron injection into, and trapping by, the plasma wave. Various approaches for injection have been proposed. Self-injection via transverse wave breaking [4] is attractive in its simplicity, however experimental reproducibility is extremely challenging. Thus, although stable self-injection regimes have been observed [5], control over the bunch properties is typically very limited. In the colliding pulse scheme, a beat-wave between the main laser-pulse driving the plasma-wave, and an additional counterpropagating pulse, heats some of the electrons at the collision point such that they gain sufficient longitudinal momentum to be trapped [6,7]. Alternatively,

*Robbie.Scott@stfc.ac.uk

Published by the American Physical Society under the terms of the Creative Commons Attribution 4.0 International license. Further distribution of this work must maintain attribution to the author(s) and the published article's title, journal citation, and DOI. ionization injection [8-12], uses gas mixtures with different ionization thresholds to inject electrons that are liberated in the correct phase of the plasma wave. Altering the plasma density profile offers another way to control electron trapping. In slow density down-ramps [4,13-17], the phase velocity of the plasma wave behind the laser-pulse is reduced, meaning a proportion of the plasma wave's electrons are faster than the local phase velocity, so they can propagate into the accelerating region of the wake and become trapped.

Sharp density down-ramps with a scale length less than the plasma wavelength can also induce injection, but through a different mechanism $[18,19]$. This has been extensively investigated theoretically [18-21]. Recent work $[22,23]$ has successfully used flow discontinuities in supersonic flows to induce a shock front, providing the sharp density transition required for injection.

Another body of work used blast-wave structures to induce a sharp density down ramp. In this method, either another laser or a prepulse heats the plasma which then expands, forming a blast-wave. The blast-wave is ellipsiodal in shape and propagates principally radially with respect to the heating laser's axis. Consequently the region on axis has a density below that of the background plasma (Fig. 3). When another laser is employed, the blast-wave is aligned at an angle to the main pulse [24-26], in these 
experiments enhancement in the number of injected electrons, their transverse emittance and the ability to generate monoenergetic beams was observed. Alternatively, in the case of a prepulse, the blast-wave is aligned with the axis of the main laser pulse [27-30]. This is the focus of our work. Experimental and theoretical studies [27-30] have shown that a laser prepulse aligned on-axis significantly improved the divergence of the accelerated electrons, and was able to produce quasi-monoenergetic electron beams when the blast-wave was present. In these previous works, the improvement has been attributed to two mechanisms which have a density dependance: below a background number density of $\sim 3 \times 10^{19} \mathrm{~cm}^{-3}$, sharp-density down-ramp injection at the leading edge is found to be the dominant mechanism [27,28]. At higher densities $\left(\geq 3 \times 10^{19} \mathrm{~cm}^{-3}\right)$ the properties of the electron bunch were found to be improved by the presence of the short plasma channel created by the prepulse [31-34]. The addition of a $\sim 0.2 \mathrm{~T}$ magnetic field reduced the plasma channel radial expansion rate, improving laser guiding, and reducing the electron beam divergence $[29,35,36]$.

In this paper we examine the role of a coaxial, prepulseinduced, cylindrical blast-wave on injection and acceleration. Due to the low density in our setup (and in agreement with Hosokia et al [28]), we find that laser-pulse guiding within the blast-wave structure has no beneficial effect. Importantly, and for the first time, we show that the electrons undergo a two-stage acceleration mechanism: first, a wakefield is generated by the laser within the homogenous plasma preceding the blast-wave. As this enters the density downramp caused by the blast-wave, injection and modest acceleration occurs. A secondary wakefield is then generated after the laser has passed the blast-wave structure, the preaccelerated electrons encounter this secondary wakefield and are then accelerated to higher energies. We show that this two-stage injection and acceleration process is beneficial in three respects; increasing the total charge injected, reducing the bunch transverse size, and reducing the transverse momentum of those electrons which are trapped. Furthermore, due to the efficiency of the trapping process, electrons can be accelerated in multiple buckets behind the drive laser.

\section{EXPERIMENTAL SETUP}

Experiments on the effect of prepulse-induced blastwaves were performed on the Astra-Gemini laser at the Rutherford Appleton Laboratory. This system delivers $45 \mathrm{fs,}$ $440 \mathrm{~mJ}, 800 \mathrm{~nm}$ laser pulses on target at $5 \mathrm{~Hz}$. An $f / 7$ offaxis parabolic mirror focusses to a spot of $10 \mu \mathrm{m}$ FWHM at the center of a gas cell. The maximum incident peak power, focal intensity and normalized vector potential were approximately $P=9.8 \mathrm{TW}, \mathrm{I}=8.6 \times 10^{18} \mathrm{~W} \mathrm{~cm}^{-2}$, and $a_{0}=$ 2.1 respectively. The gas cell was a $15 \mathrm{~mm}$ long, $300 \mu \mathrm{m}$ diameter capillary [37] filled with hydrogen gas (H) doped with $4 \%$ nitrogen $(\mathrm{N})$.
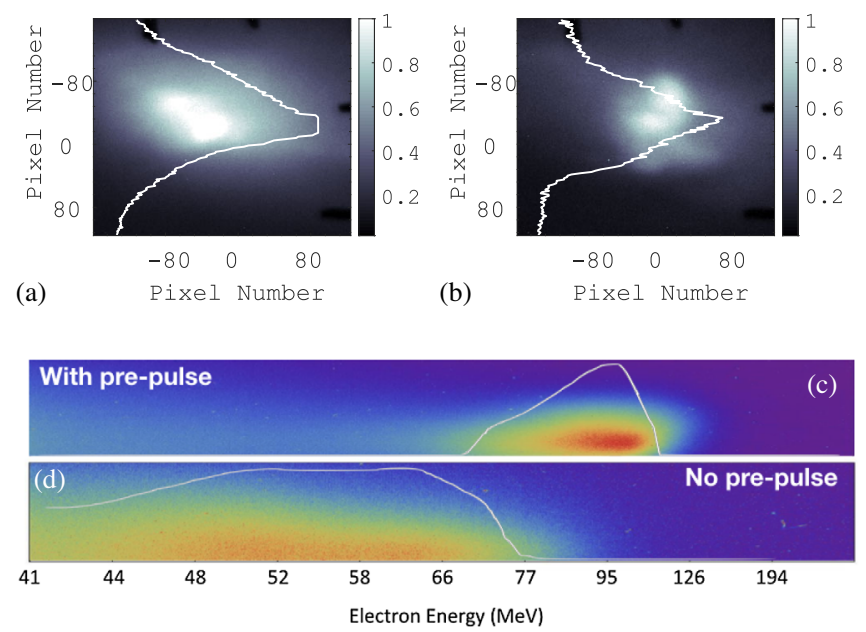

FIG. 1. Electron beam energy deposition on a lanex screen, indicative of beam divergence (a) with prepulse, and (b) without. Note (a) is unfortunately saturated. Lines depict the relative signal and show both increased total signal (charge) and reduced divergence. Representative electron spectra with prepulse (c) and without (d). Spectra are imaged in the vertical direction corroborating (a) and (b).

Unwanted reflections caused a prepulse with a contrast ratio of $1 \times 10^{-5}$ (intensity $8.6 \times 10^{13} \mathrm{~W} \mathrm{~cm}^{-2}$ ), at $\sim 65 \mathrm{ps}$ before the main pulse. The prepulse was removed using optics with better antireflection coatings. As shown in Fig. 1, when the prepulse was present, enhanced electron beams were observed, these exhibited increased charge (by $\sim 1.5 \times$ ) and a reduction in the beam divergence (from $\sim 22$ to $12 \mathrm{mrad}$ ) at a pressure of $200 \mathrm{mbar}$. While the advantages of these properties were clear, the underlying physical mechanisms were not.

\section{SIMULATIONS}

In order to better understand the physics underlying the experimental observations, numerical modeling was performed of the effects of the prepulse, the subsequent plasma evolution, the high intensity laser-plasma interaction and subsequent wakefield acceleration. Due to the disparate temporal scales of the experimental interaction-the initial prepulse occurs $65 \mathrm{ps}$ before the main interaction-in combination with the need for a kinetic treatment for the interaction of the highest intensity pulse, a chain of codes was employed. The simulation methodology and associated physics are described below.

The interaction of the laser prepulse with the underdense gas was modeled in 2 dimensions (D) in Cartesian coordinates, using the EPOCH particle-in-cell code [38]. Laser intensities, $\mathrm{f} / \#$, gas densities etc., were all as-per the experiment. Simulation parameters were: 20 electrons and 20 ions per cell, the moving window simulation box was $150 \times 120 \mu \mathrm{m}$, and the grid resolution $0.04 \mu \mathrm{m}$. Both $\mathrm{H}$ and $\mathrm{N}$ species were initialized unionized at $2 \mathrm{eV}$, and 


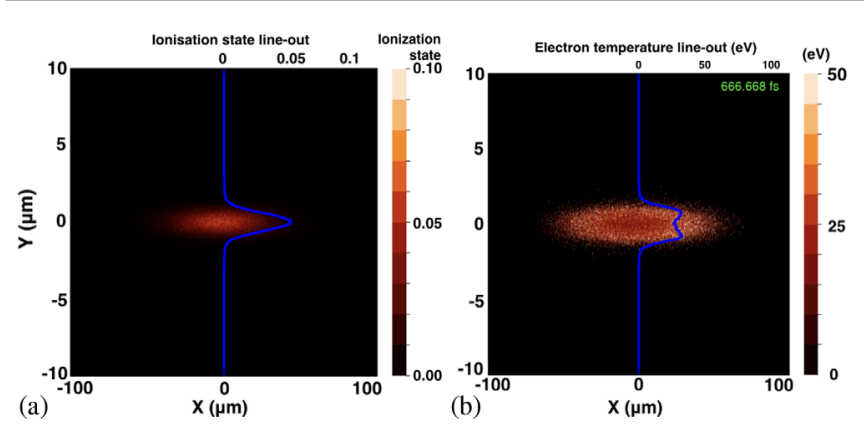

FIG. 2. (a) EPOCH simulations of hydrogen ionization caused by the prepulse. The blue line is an ionization fraction line-out along $X=0$ (upper $\mathrm{X}$-axis). (b) Electron temperatures. Here the blue line is an electron temperature line-out along $X=0$ (upper $\mathrm{X}$-axis).

multiphoton, tunneling, and field ionization processes were modelled [38]. The initial temperature was chosen to minimize numerical heating, it did not affect the initial ionization level, which remained zero until the laser pulse arrived. At the prepulse intensity, the tunneling and field ionization rates are negligible, however the threshold for multiphoton ionization (MPI) is exceeded, creating a thin filament of partially ionized plasma on-axis [see Fig. 2(a)]. In EPOCH MPI ionization rates are based on a cross section for the absorption of multiple photons [38]. This is based on a semiempirical WKB model by Ammosov et al. [39] which treats the n-photon ionization of the atom as a hydrogenlike series of excited Rydberg states, and is a reasonable approximation for the excited states of low- $Z$ atoms. Since the intensity threshold for ionization of $\mathrm{H}$ was only exceeded near the peak laser focus, the filament generated had a radius less than the laser focal spot size $w_{0}$, and a length of order the Rayleigh range $Z_{R}=\pi w_{0}^{2} / \lambda$. MPI imparts momentum to the ionized electrons [40], combined with ponderomotive effects, this results in a free electron population with a higher temperature than that of the cold background ions [Fig. 2(b)].

The evolution of the preplasma in the $65 \mathrm{ps}$ interval between the pre and main laser pulses was modeled by mapping the 2D ion number, charge state, electron and ion temperature distributions from EPOCH into radiationhydrodynamics simulations.

Assuming cylindrical symmetry, 1D Hyades [41] and 2D FLASH [42] simulations of the electron-ion equilibration process and the resultant hydrodynamic motion were performed. It was found that the electrons rapidly thermalize with the ions, locally heating the ions, creating a Gaussian spatial distribution in ion temperature, and hence ion pressure. The ion pressure distribution in turn drives a shock outwards, predominantly in the radial direction from the filament axis. This forms a characteristic cylindrical blast-wave structure; a shock front, followed by a density depression below that of the background plasma, as depicted in Figs. 3(a) and (b).
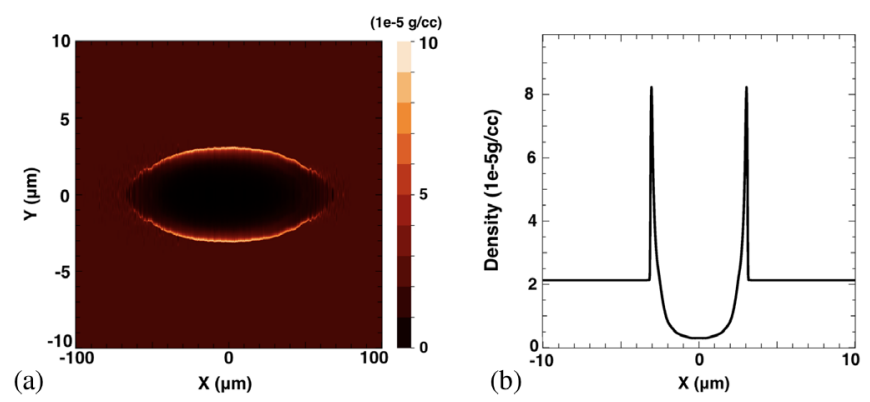

FIG. 3. (a) The blast-wave structure which evolves from the initial electron temperature profile induced by the laser pre-pulse. (b) A $1 \mathrm{D}$ slice of the density profile at $X=0$, showing the characteristic blast-wave structure. Note this figure has been labelled with $\mathrm{X}$ and $\mathrm{Y}$ for consistency with the $\mathrm{EPOCH}$ simulations, in these simulations $\mathrm{Y}$ is in fact the radial dimension in the cylindrical coordinate system employed.

After 65 ps 2D temperature and density maps from the FLASH radiation-hydrodynamics simulation were mapped back into EPOCH in order to understand the effects of the blast-wave induced density and temperature profiles on the main high-intensity $\left(8.6 \times 10^{18} \mathrm{~W} \mathrm{~cm}^{2}\right)$ laser-plasma interaction. Two scenarios were investigated: (1) a uniform density distribution, replicating the situation where no prepulse is present; (2) the presence of a prepulse-induced blast-wave centered at the laser focus, as shown in Fig. 3(a). This was mapped from the 2D radiation-hydrodynamics runs, and is our best representation of the effect of the prepulse on the background plasma. Aside from the addition of the blast-wave in one simulation, the initial conditions of the two EPOCH simulations were identical.

\section{DISCUSSION}

In the absence of a prepulse, the main high-intensity laser propagates through the uniform underdense plasma, creating a wakefield behind the laser pulse. Importantly, in the case with no prepulse, no electrons were trapped (defined as those which reached energies above $1 \mathrm{MeV}$ ), indicating the laser intensity was insufficient for nitrogen ionization injection to occur. In contrast, when the main laser pulse interacts with the blast-wave created by the prepulse (Fig. 3), copious trapping is observed as the wake enters the blast-wave structure; the wake encounters a sudden reduction in the background plasma density (Fig. 4), and hence electron density, causing injection via sharp-density down-ramp injection (wake wavelength $\sim 7 \mu \mathrm{m}$, density scalelength $\sim 1 \mu \mathrm{m})$. The trapped electrons are subsequently accelerated as the laser pulse and following wake propagates into the blast-wave structure down the density profile within the blast-wave's "nose." For our conditions, after $\sim 30 \mu \mathrm{m}$ of propagation within the blastwave structure, the electrons reach the longitudinal densityfloor of the blast-wave and dephase with the wakefield. By this point the electrons have gained a modest amount of 

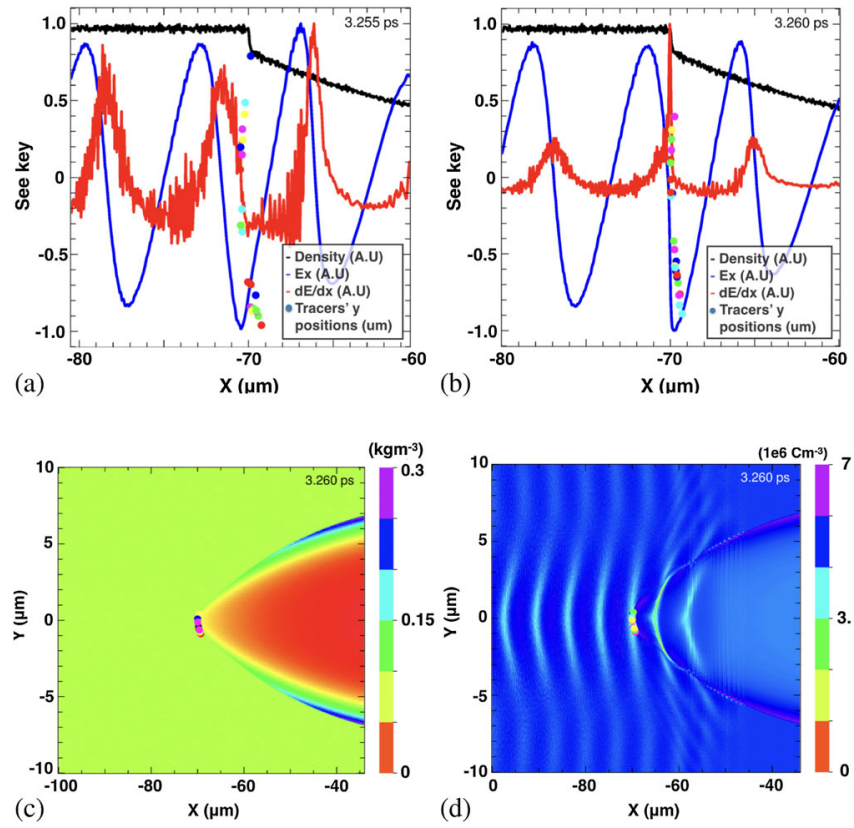

FIG. 4. (a) Lineouts along $y=0$, just before trapping occurs: mass density (black), electric field in X (blue), electric field gradient in X (red), the markers show the positions of the tracer particles, for these, the Yaxis is position. (b) The same graph as (a) but at the time when the wake enters the blast-wave, and trapping occurs. The density drop induces a sharp peak in the electric field gradient, which traps the particles. (c) The mass density at the time of injection, markers show the tracer positions. (d) Charge density showing the wake structures and tracer positions.

kinetic energy $\sim 1.5-2 \mathrm{MeV}$. After their initial acceleration, the electron bunch(es) are relatively unaffected during their propagation through the blast-wave structure, and exit it with kinetic energies in the range $1.75-2.25 \mathrm{MeV}$.

On passing the blast-wave, the main laser pulse drives a new wakefield in the uniform plasma beyond the blast-wave [Fig. 5(a)]. The $\sim 2 \mathrm{MeV}$ preaccelerated electron bunch(es) are easily retrapped in this new wakefield and are accelerated to higher energies [Fig. 5(b)].

Two aspects of this injection mechanism are of particular note: first, trapping at the entrance to the blast-wave is not limited to the first or second wakefield period trailing the laser, instead electron bunches are trapped in several wakefield buckets as they enter the blast-wave. This results in a train of preaccelerated electrons which, on exiting the blast-wave structure, are trapped and accelerated in multiple wakefield buckets in the wakefield driven beyond the blast-wave. In the simulations presented here, at least 13 trapped electron bunches were observed [Fig. 5(b)]. While the energies at this point are modest $(\sim 30 \mathrm{MeV})$, acceleration beyond the blast-wave was only tracked in the simulations over a distance of $\sim 80 \mu \mathrm{m}$; there is no apparent reason why these electrons could not be accelerated to energies obtainable by other injection mechanisms. The width of the quasi-monoenergetic peak was $8 \mathrm{MeV}(\Delta E / E=25 \%)$.
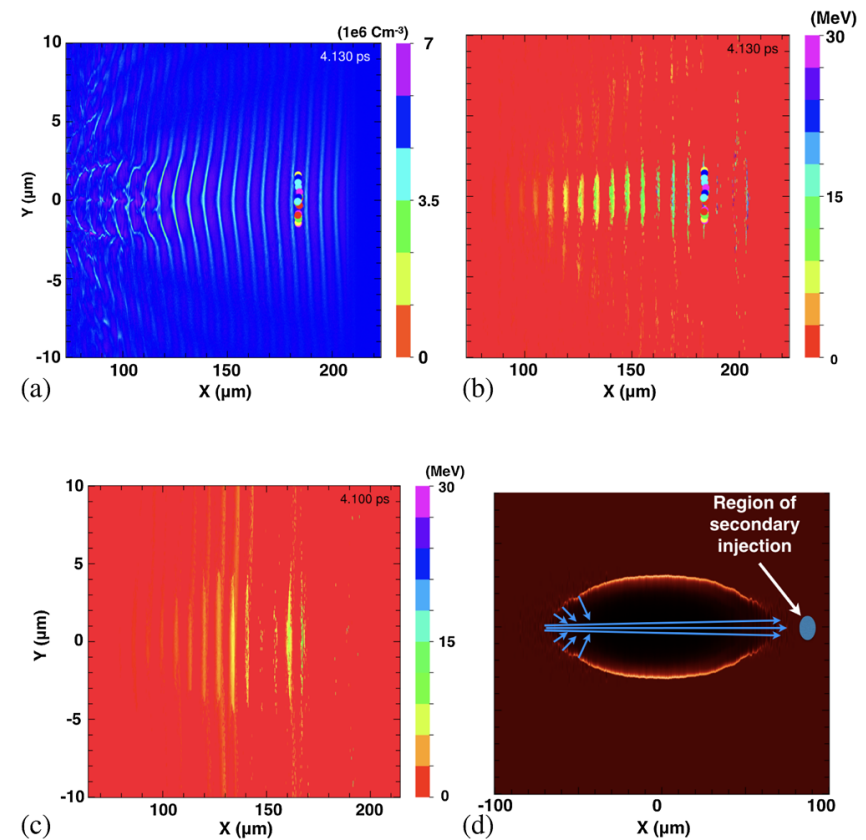

FIG. 5. Plasma parameters after the laser has propagated to $X=205 \mu \mathrm{m}$. The blast-wave ends at $X=70 \mu \mathrm{m}$. (a) The charge density distribution shows the wakefield structures created after the blast-wave; these wakes trap those electrons which are preaccelerated within the blast-wave. (b) This plot of cellaveraged particle kinetic energy shows multiple bunches are accelerated to higher energies by the multiple wakes trailing the laser. The laser is not shown, but is centered at $X=205 \mu \mathrm{m}$. Only those electrons originating from the nitrogen atoms are shown. (c) As per (b) but with a density profile which only varies as a function of $x$. Here the lateral extent of the bunches is significantly increased from $\sim 2 \mu \mathrm{m}$ to $10 \mu \mathrm{m}$. (d) Blue arrows illustrate the initial propagation directions of those electrons injected at the blast-wave. The electrons are injected approximately perpendicularly to the blast-wave at a given location, so only those with small divergence (near $y=0$ ) enter the region of secondary injection.

The second notable aspect of this interaction is that the blast-wave has the potential to act as a "phase-space filter" as, due to the blast-wave's axial length, only those electrons with sufficiently small transverse velocity after initial injection at the blast-wave nose enter into the appropriate region of the secondary wakefield for secondary injection/ acceleration (Fig. 5(d)). This is explained as follows: as the wakes enter the blast-wave structure, the simulations reveal that injection occurs all along the blast-wave's leading-edge (as viewed by the incoming wakes). The electrons are accelerated parallel to the wake's local electric field vector. Due to the curvature of the wake entering the blast-wave this means that the transverse velocity of the electrons trapped at the nose of the blast-wave is a function of the electron's initial radial distance from the laser's axis. Consequently, only those electrons which were initially on, or very near, the axis have sufficiently small transverse velocity to arrive near the center of the secondary wake 
(having propagated along the length of, and subsequently exited, the blast-wave structure) - large transverse velocity would mean that they arrive at the second trapping point at a large transverse distance from the axis, where the wakefields are weaker, precluding trapping from occurring. Therefore only those electrons with sufficiently small transverse velocity are re-injected into the secondary wake, trapped, and accelerated to high energy.

As a consequence of this "phase-space filtering" mechanism, the axial length of the blast-wave determines the transverse velocity of those electrons which will be postaccelerated to higher energies. It is notable that were the blast-wave to be made progressively longer (e.g., by changing the focussing optics), those electrons which receive a secondary acceleration after the blast-wave will, at the point of re-injection, have concomitantly smaller transverse velocities.

A secondary consequence of the highly curved blast-wave nose and the resulting filtering process is that, at the point of reinjection into the secondary wake, the retrapped electron bunches have a smaller transverse size, than would be the case were it less curved. A zero curvature scenario was investigated by replacing the 2D blast-wave structure [den$\operatorname{sity}(\mathrm{x}, \mathrm{y})]$ with a structure which only varies as a function of the $x$ direction [density $(x)]$ : the profile shown in Fig. 3(a) at $y=0$, was mapped to all y-coordinates. This new density distribution exhibited approximately the same trapping and injection characteristics as those of the 2D structure, but notably in this case, the transverse size of the bunches are significantly extended to $\sim 10 \mu \mathrm{m}$ [Fig. 5(c)]. This verifies the importance of the 3D shape of the blast-wave structure, which acts to only select those electrons initially injected with a small transverse velocity. To our knowledge, this is the first report of the use of a transversely shaped density ramp to reduce the transverse bunch size and emittance of the injected bunch. The prepulse improved emittance both experimentally and in simulations (in both cases from $\sim 20 \mathrm{mrad}$ to 10), however no attempt was made to optimize emittance here through extension of the blast-wave region.

\section{CONLUSIONS}

In summary we have shown that a prepulse can create an ionized plasma filament through multiphoton ionization, this heats the electrons, which in turn equilibrate with the cold background ions, creating a pressure distribution which drives a blast-wave. The blast-wave forms an ellipsoidal density structure which is, by its nature, aligned with the trailing drive pulse. Down-ramp injection and trapping at the leading edge of the blast-wave structure leads to acceleration of electrons to energies of $\sim 2 \mathrm{MeV}$. For the first time, we have shown that these electrons are injected into, retrapped, and accelerated by the wakefields driven beyond the blast-wave structure. Furthermore, due to the longitudinal extent of the blast-wave, only those electrons with small transverse velocity are retrapped and further accelerated. With the appropriate focusing optics, this has the potential to reduce the initial bunch size and emittance of the reaccelerated electrons. Finally, due to the efficiency of this injection mechanism, this secondary-acceleration can occur in multiple "buckets" of the wakes trailing the laser.

Prepulse induced blast-wave injection appears to provide a simple, robust, injection mechanism for generation of high charge, reduced emittance electron bunches. We note that the collimating effect of the blast-wave could be further optimized by adjusting the f/\# of the focusing optic and/or using a prepulse of a different wavelength.

\section{ACKNOWLEDGMENTS}

This work was supported by the STFC and EPSRC (Grants No. EP/G054950/1, No. EP/G056803/1, No. EP/ G055165/1, No. EP/L000237/1, No. EP/H011145/1, No. EP/G067791/1, No. 1093881) and HGF VI Helmholtz Association VH-VI-503.

[1] S. P. D. Mangles, C. D. Murphy, Z. Najmudin, A. G. R. Thomas, J. L. Collier, A. E. Dangor, E. J. Divall, P. S. Foster, J. G. Gallacher, C. J. Hooker, D. A. Jaroszynski, A. J. Langley, W. B. Mori, P. A. Norreys, F. S. Tsung, R. Viskup, B. R. Walton, and K. Krushelnick, Nature (London) 431, 535 (2004).

[2] C. G. R. Geddes, C. Toth, J. van Tilborg, E. Esarey, C. B. Schroeder, D. Bruhwiler, C. Nieter, J. Cary, and W. P. Leemans, Nature (London) 431, 538 (2004).

[3] J. Faure, Y. Glinec, A. Pukhov, S. Kiselev, S. Gordienko, E. Lefebvre, J. P. Rousseau, F. Burgy, and V. Malka, Nature (London) 431, 541 (2004).

[4] S. Bulanov, N. Naumova, F. Pegoraro, and J. Sakai, Phys. Rev. E 58, R5257 (1998).

[5] J. Osterhoff, A. Popp, Z. Major, B. Marx, T. P. RowlandsRees, M. Fuchs, M. Geissler, R. Hörlein, B. Hidding, S. Becker, E. A. Peralta, U. Schramm, F. Grüner, D. Habs, F. Krausz, S. M. Hooker, and S. Karsch, Phys. Rev. Lett. 101, 085002 (2008).

[6] E. Esarey, R. F. Hubbard, W. P. Leemans, A. Ting, and P. Sprangle, Phys. Rev. Lett. 79, 2682 (1997).

[7] J. Faure, C. Rechatin, A. Norlin, A. Lifschitz, Y. Glinec, and V. Malka, Nature (London) 444, 737 (2006).

[8] D. Umstadter, J. K. Kim, and E. Dodd, Phys. Rev. Lett. 76, 2073 (1996).

[9] T. P. Rowlands-Rees, C. Kamperidis, S. Kneip, A. J. Gonsalves, S. P. D. Mangles, J. G. Gallacher, E. Brunetti, T. Ibbotson, C. D. Murphy, P. S. Foster, M. J. V. Streeter, F. Budde, P. A. Norreys, D. A. Jaroszynski, K. Krushelnick, Z. Najmudin, and S. M. Hooker, Phys. Rev. Lett. 100, 105005 (2008).

[10] A. Pak, K. A. Marsh, S. F. Martins, W. Lu, W. B. Mori, and C. Joshi, Phys. Rev. Lett. 104, 025003 (2010).

[11] C. McGuffey, A. G. R. Thomas, W. Schumaker, T. Matsuoka, V. Chvykov, F. J. Dollar, G. Kalintchenko, V. 
Yanovsky, A. Maksimchuk, K. Krushelnick et al., Phys. Rev. Lett. 104, 025004 (2010).

[12] N. Bourgeois, J. Cowley, and S. M. Hooker, Phys. Rev. Lett. 111, 155004 (2013).

[13] C. G. R. Geddes, K. Nakamura, G. R. Plateau, C. Toth, E. Cormier-Michel, E. Esarey, C. B. Schroeder, J. R. Cary, and W. P. Leemans, Phys. Rev. Lett. 100, 215004 (2008).

[14] R. M. G. M. Trines, R. Bingham, Z. Najmudin, S. Mangles, L. O. Silva, R. Fonseca, and P. A. Norreys, New J. Phys. 12, 045027 (2010).

[15] A. J. Gonsalves, K. Nakamura, C. Lin, D. Panasenko, S. Shiraishi, T. Sokollik, C. Benedetti, C. B. Schroeder, C. G. R. Geddes, J. van Tilborg, J. Osterhoff, E. Esarey, C. Toth, and W. P. Leemans, Nat. Phys. 7, 862 (2011).

[16] R. Weingartner, S. Raith, A. Popp, S. Chou, J. Wenz, K. Khrennikov, M. Heigoldt, A. R. Maier, N. Kajumba, M. Fuchs, B. Zeitler, F. Krausz, S. Karsch, and F. Grüner, Phys. Rev. Accel. Beams 15, 111302 (2012).

[17] M. Hansson, T. L. Audet, H. Ekerfelt, B. Aurand, I. G. González, F. G. Desforges, X. Davoine, A. Maitrallain, S. Reymond, P. Monot, A. Persson, S. D. Dufrénoy, C.-G. Wahlström, B. Cros, and O. Lundh, Plasma Phys. Controlled Fusion 58, 055009 (2016).

[18] H. Suk, N. Barov, J. B. Rosenzweig, and E. Esarey, Phys. Rev. Lett. 86, 1011 (2001).

[19] H. Suk, C. Kim, G. Kim, J. Kim, I. Ko, and H. Lee, Phys. Lett. A 316, 233 (2003).

[20] R. J. England, J. B. Rosenzweig, and N. Barov, Phys. Rev. E 66, 016501 (2002).

[21] A. V. Brantov, T. Z. Esirkepov, M. Kando, H. Kotaki, V. Y. Bychenkov, and S. V. Bulanov, Phys. Plasmas 15, 073111 (2008).

[22] K. Schmid, A. Buck, C. M. S. Sears, J. M. Mikhailova, R. Tautz, D. Herrmann, M. Geissler, F. Krausz, and L. Veisz, Phys. Rev. Accel. Beams 13, 091301 (2010).

[23] A. Buck, J. Wenz, J. Xu, K. Khrennikov, K. Schmid, M. Heigoldt, J. M. Mikhailova, M. Geissler, B. Shen, F. Krausz, S. Karsch, and L. Veisz, Phys. Rev. Lett. 110, 185006 (2013).

[24] J. U. Kim, N. Hafz, and H. Suk, Phys. Rev. E 69, 026409 (2004).

[25] T.-Y. Chien, C.-L. Chang, C.-H. Lee, J.-Y. Lin, J. Wang, and S.-Y. Chen, Phys. Rev. Lett. 94, 115003 (2005).

[26] J. Faure, C. Rechatin, O. Lundh, L. Ammoura, and V. Malka, Phys. Plasmas 17, 083107 (2010).
[27] T. Hosokai, K. Kinoshita, A. Zhidkov, K. Nakamura, T. Watanabe, T. Ueda, H. Kotaki, M. Kando, K. Nakajima, and M. Uesaka, Phys. Rev. E 67, 036407 (2003).

[28] T. Hosokai, K. Kinoshita, A. Zhidkov, K. Nakamura, H. Kotaki, M. Kando, K. Nakajima, and M. Uesaka, Phys. Plasmas 11, L57 (2004).

[29] T. Hosokai, K. Kinoshita, A. Zhidkov, A. Maekawa, A. Yamazaki, and M. Uesaka, Phys. Rev. Lett. 97, 075004 (2006).

[30] T. Hosokai, K. Kinoshita, T. Ohkubo, A. Maekawa, M. Uesaka, A. Zhidkov, A. Yamazaki, H. Kotaki, M. Kando, K. Nakajima, S. V. Bulanov, P. Tomassini, A. Giulietti, and D. Giulietti, Phys. Rev. E 73, 036407 (2006).

[31] C. G. Durfee and H. M. Milchberg, Phys. Rev. Lett. 71, 2409 (1993).

[32] T. R. Clark and H. M. Milchberg, Phys. Rev. Lett. 78, 2373 (1997).

[33] T. R. Clark and H. M. Milchberg, Phys. Rev. E 61, 1954 (2000).

[34] P. Sprangle, B. Hafizi, J. R. Peñano, R. F. Hubbard, A. Ting, C. I. Moore, D. F. Gordon, A. Zigler, D. Kaganovich, and T. M. Antonsen, Phys. Rev. E 63, 056405 (2001).

[35] N. Nakanii, T. Hosokai, K. Iwasa, S. Masuda, A. Zhidkov, N. Pathak, H. Nakahara, Y. Mizuta, N. Takeguchi, and R. Kodama, Phys. Rev. Accel. Beams 18, 021303 (2015).

[36] T. Hosokai, A. Zhidkov, A. Yamazaki, Y. Mizuta, M. Uesaka, and R. Kodama, Appl. Phys. Lett. 96, 121501 (2010).

[37] J. Osterhoff, A. Popp, Z. Major, B. Marx, T. P. RowlandsRees, M. Fuchs, M. Geissler, R. Hörlein, B. Hidding, S. Becker et al., Phys. Rev. Lett. 101, 085002 (2008).

[38] T. D. Arber, K. Bennett, C. S. Brady, A. Lawrence-Douglas, M. G. Ramsay, N. J. Sircombe, P. Gillies, R. G. Evans, H. Schmitz, A. R. Bell, and C. P. Ridgers, Plasma Phys. Controlled Fusion 57, 113001 (2015).

[39] N. Delone and V. Kranov, Multiphoton Processes in Atoms (Springer Verlag, Berlin, 2000), Vol. 13.

[40] G. Mainfray and G. Manus, Rep. Prog. Phys. 54, 1333 (1991).

[41] J. T. Larsen and S. M. Lane, J. Quant. Spectroscopy Radiat. Transfer 51, 179 (1994), special Issue Radiative Properties of Hot Dense Matter.

[42] B. Fryxell, K. Olson, P. Ricker, F. X. Timmes, M. Zingale, D. Q. Lamb, P. MacNeice, R. Rosner, J. W. Truran, and H. Tufo, Astrophys. J. Suppl. Ser. 131, 273 (2000). 